\title{
Caracterização geoquímica molecular de arenitos asfálticos a partir da desagregação branda de asfaltenos
}

\section{Fernanda Lins da Costa ${ }^{1}$ \\ Laercio Lopes Martins ${ }^{1}$ \\ Georgiana Feitosa da Cruz ${ }^{1 *}$ \\ Lívia Carvalho Santos ${ }^{1}$ \\ Alexsandro Araujo da Silva ${ }^{2}$}

'Laboratório de Engenharia e Exploração de Petróleo da Universidade Estadual do Norte Fluminense (LENEP/UENF) - Macaé (RJ), Brasil.

${ }^{2}$ Central Analítica Fernanda Coutinho, Instituto de Química da Universidade do Estado do Rio de Janeiro (UERJ) - Rio de Janeiro (RJ), Brasil.

*Autor correspondente: geofec@gmail.com

\section{Resumo}

Uma quantidade significativa de hidrocarbonetos, provavelmente remanescentes do "óleo original", fica retido (ocluído) no interior da matriz de asfaltenos e protegido dos processos de alteração secundária que ocorreram posteriormente nos reservatórios de petróleo. Neste trabalho fez-se uma reação de oxidação dos asfaltenos usando $\mathrm{NaIO}_{4}-\mathrm{NaH}_{2} \mathrm{PO}_{4} \mathrm{em}$ amostras de arenitos asfálticos, com o intuito de liberar os hidrocarbonetos ocluídos e recuperar a informação geoquímica molecular original dessas amostras fortemente degradadas por processos pós-deposicionais. Os resultados obtidos a partir da análise SARA e do monitoramento seletivo dos íons-fragmentos em $\mathrm{m} / z$ 85, 177, 191 e 217 revelaram que os componentes do petróleo estavam protegidos pela estrutura asfaltênica e que a reação de oxidação foi eficiente na sua liberação. Além disso, observou-se que a severa biodegradação presente nos maltenos livres e ausente nos ocluídos interfere e mascara os parâmetros geoquímicos. Portanto, concluiu-se que o estudo dos biomarcadores ocluídos em estruturas asfaltênicas é uma ferramenta valiosa na interpretação de parâmetros geoquímicos para caracterização de petróleos, visto que estes são remanescentes do "óleo original".

Palavras-chave: arenitos asfálticos, biodegradação, asfaltenos, biomarcadores ocluídos.

\section{Abstract}

A significant quantity of hydrocarbons, probably remnants of the "original oil", is retained (occluded) within the asphaltene matrix and protected from the secondary alteration processes that occurred subsequently in the petroleum reservoirs. In this work, it was done an oxidation reaction of asphaltenes using $\mathrm{NaIO}_{4}-\mathrm{NaH}_{2} \mathrm{PO}_{4}$ in samples of tar sands in order to release the occluded hydrocarbons and recover the original molecular geochemical information of these samples heavily degraded by post-depositional processes. The results obtained from the SARA's and selective fragments-ions monitoring into $\mathrm{m} / \mathrm{z} 85,177,191$ and 217 revealed that the components of petroleum were protected by asphaltene structure and the reaction of oxidation was efficient in this release. Moreover, it was observed that severe biodegradation present in the free malthenes and absent at occluded, interferes and mask the geochemical parameters. Therefore, it was concluded that occluded biomarkers study in asphaltene structures is a valuable tool in the interpretation of geochemical parameters for petroleum characterization since these are remainings of the original oil.

Keywords: tar sands, biodegradation, asphaltenes, occluded biomarkers. 


\section{INTRODUÇÃO}

O petróleo é constituído por uma fração de maltenos que inclui hidrocarbonetos (HCs) saturados, aromáticos e resinas, e por uma fração de asfaltenos, que são macromoléculas de alto peso molecular (Speight 1994, Mansoori 1997, Calemma et al. 1998, Gawrys \& Kilpatrick 2005) definidos em relação à sua solubilidade (Gürgey 1998, Seidl et al. 2004, Badre et al. 2006). Os asfaltenos são insolúveis em $n$-alcanos de baixa massa molecular, como $n$-pentano, $n$-hexano ou $n$-heptano, e solúveis em tolueno ou benzeno (Mansoori 1997, Calemma et al. 1998).

O estudo geoquímico molecular das frações do petróleo, baseado na análise de biomarcadores, auxilia na identificação e avaliação das rochas geradoras, no melhor entendimento sobre os processos que envolvem sua deposição, formação e preservação da matéria orgânica, além da geração e migração do óleo, visando orientar a exploração para áreas mais favoráveis à existência de acumulações comerciais de óleo e gás. É possível também, por meio da análise de biomarcadores, estudar e avaliar os processos secundários que ocorrem após o óleo ter sido acumulado no reservatório.

Para uma análise molecular mais adequada para fins de exploração, é conveniente fazer a separação de maltenos e asfaltenos, normalmente por meio da precipitação com solvente. No entanto, devido à sua natureza polar e coloidal, os asfaltenos podem absorver e/ou adsorver algumas das moléculas presentes na fração maltênica, formando aglomerados ("gaiolas") com essas moléculas ocluídas (Gürgey 1998, Azevedo et al. 2009). Devido a isso, a separação das frações normalmente não é completa e pode ocorrer distorção na interpretação das informações geoquímicas obtidas a partir da análise de hidrocarbonetos saturados e aromáticos, incluindo os biomarcadores. Então, se os perfis de biomarcadores podem ser alterados pelo processo de precipitação de asfaltenos, os estudos de correlação óleo-óleo e óleo-rocha geradora, avaliações dos ambientes paleodeposicionais, da maturidade, da biodegradação e de outros parâmetros geoquímicos importantes para a exploração (Mackenzie 1984, Moldowan et al. 1985), também podem ser comprometidos (Gürgey 1998). Além disso, o petróleo ainda está suscetível a diversas alterações pós-acumulação no reservatório (Tissot \& Welte 1984). A variabilidade que essas alterações provocam nas características físico-químicas ocorre devido à biodegradação, lavagem por águas meteóricas (water washing), alteração térmica, desasfaltamento ou segregação gravitacional. Assim, o escoamento de hidrocarbonetos do reservatório ou a adição deles a partir das rochas geradoras ativas pode modificar o óleo original.

A circulação de águas meteóricas transforma os óleos leves em óleos pesados e a alteração térmica os leva a condensados e gás, acompanhados do desasfaltamento natural e da formação do pirobetume no reservatório. Todos estes fatores influenciam na economia de uma descoberta de petróleo, sendo então de grande importância entender e prever esses processos para estimar o valor do petróleo que pode ocorrer em um prospecto (Hunt 1996).
Dentre esses processos de alteração secundária do petróleo, a biodegradação ganha destaque por ser o processo de alteração secundária mais importante e devido ao volume de óleo pesado que tem sido explorado ao redor do mundo. Esta alteração é atribuída à ação de micro-organismos distintos (Milner et al. 1977, Connan 1984, Palmer 1983, Blanc \& Connan 1994, Peters et al. 2005, Da Cruz et al. 2008, Da Cruz et al. 2011) e está vinculada a determinadas condições geológicas e geoquímicas que permitem melhorar a vida microbiana, tais como as existentes na interface óleo-água em um reservatório de petróleo (Larter et al. 2006, Da Cruz \& Marsaioli 2012). Segundo Peters et al. (2005), as condições ótimas estão diretamente relacionadas ao fácil acesso ao petróleo e nutrientes inorgânicos (como o fósforo e traços de metais) presentes em reservatórios com temperaturas inferiores a $80^{\circ} \mathrm{C}$, que são condições essenciais para a sobrevivência dos micro-organismos. Além disso, é necessário que a rocha tenha permeabilidade e porosidade suficientes para permitir a difusão dos nutrientes e que a salinidade da água da formação não ultrapasse o limite de 150 ppm. Associado a todas essas condições, é imprescindível a presença dos micro-organismos biodegradadores e a ausência dos não-biodegradadores de hidrocarbonetos, os quais são considerados como "venenos naturais", visto que são tolerantes às condições dos reservatórios e inibem e/ou limitam o crescimento e atividade enzimática dos micro-organismos degradadores (Da Cruz 2009, Da Cruz \& Marsaioli 2012).

Os efeitos mais notáveis da biodegradação envolvem a perda dos compostos mais leves, com a remoção seletiva iniciando-se preferencialmente pelos $n$-alcanos, alquilcicloexanos, seguida da remoção dos isoprenoides acíclicos e dos biomarcadores terpanos bicíclicos, esteranos, hopanos, diasteranos e esteroides aromáticos, com produção de novos compostos como produtos da biodegradação, tais como $17 \alpha$, 25-norhopanos e ácidos carboxílicos (Chosson et al. 1992, Moldowan et al. 1992, Peters \& Moldowan 1993, Da Silva 2008, De Lima et al. 2010, Da Cruz \& Marsaioli 2012).

Segundo relatos da literatura (Behar et al. 1984, Ekweozor 1984, Ekweozor 1986, Peng et al. 1997, Peng et al. 1999, Liao \& Geng 2002, Azevedo et al. 2009), as moléculas aprisionadas (ou ocluídas) nas estruturas asfaltênicas ficam protegidas e pouco afetadas pelas alterações secundárias, acreditando-se que sejam remanescentes do "óleo original", e podem, portanto, ser utilizadas para estudos geoquímicos de amostras com elevado nível de biodegradação. Com isso, pode-se ter uma interpretação confiável dos parâmetros geoquímicos utilizados para caracterizar o petróleo original.

Com o intuito de liberar os biomarcadores ocluídos, técnicas como pirólise de asfaltenos, redução química e oxidações catalisadas com rutênio já foram empregadas sem grande sucesso. Atualmente têm se utilizado reações de oxidação branda com resultados mais satisfatórios para a liberação dessas moléculas (Liao \& Geng 2002, Liao et al. 2006a, Liao et al. 2006b, Azevedo et al. 2009). A oxidação branda despolimeriza a molécula de asfalteno separando 
os grupamentos aromáticos, e as pontes que ligam estes grupamentos são convertidas em cadeias periféricas com grupos carboxílicos terminais (Azevedo et al. 2009). Assim, os hidrocarbonetos obtidos após a reação de oxidação não serão gerados pelo craqueamento das ligações (ou seja, nada da porção alifática quimicamente ligada à molécula de asfalteno), mas sim remanescentes do "óleo original" ocluídos pela molécula de asfalteno (Liao \& Geng 2002, Azevedo et al. 2009).

Como os asfaltenos são um dos componentes mais polares nos reservatórios de óleo, existem fortes interações entre suas moléculas, fazendo assim com que as mesmas se tornem susceptíveis de se agregar, mesmo em baixa concentração. Para certificar-se de que a degradação de asfaltenos pode ser realizada eficazmente, é necessário reduzir essas

\subsection{Arenitos asfálticos da Bacia do Paraná}

As ocorrências de arenitos asfálticos têm sido atribuídas ao Sistema Petrolífero Irati-Piramboia, região do Anhembi, estado de São Paulo, induzidas por maturação anômala de folhelhos betuminosos da Formação Irati, a partir de intrusões ígneas dos basaltos da Formação Serra Geral, com volume estimado de 6 milhões de barris de óleo na área centro leste de São Paulo (Thomaz Filho 2008). Os arenitos asfálticos ou tar sands são arenitos preenchidos naturalmente por hidrocarbonetos pesados, como o asfalto, tendo perdido as frações mais leves por processos secundários, como a biodegradação (Garcia et al. 2011, Martins 2013). Sua exposição em afloramentos proporciona excelente oportunidade para avaliar o modelo exploratório

\section{MATERIAIS E MÉTODOS}

Foram analisadas quatro amostras de óleo pesado separadas de rochas do reservatório coletadas nas Fazendas da Mina e Betumita, borda leste da Bacia do Paraná no estado de São Paulo. As amostras (60 g da rocha pulverizada) foram submetidas a extração em Soxhlet com $300 \mathrm{~mL}$ de $n$-pentano durante 20 horas para obtenção dos maltenos. Para extração dos asfaltenos utilizou-se uma mistura de clorofórmio:metanol $(95: 5 \mathrm{v} / \mathrm{v})$ durante 46 horas. $\mathrm{O}$ asfalteno obtido foi lavado com $n$-pentano para eliminar os possíveis interferentes que poderiam ficar adsorvidos em sua superfície. Os asfaltenos obtidos foram concentrados por evaporação do solvente, pesados e submetidos a reação de oxidação branda com $\mathrm{NaIO}_{4}$ e $\mathrm{NaH}_{2} \mathrm{PO}_{4}$ segundo metodologia descrita por Azevedo et al. (2009).

Os maltenos obtidos antes e após a reação de oxidação foram pesados e submetidos ao fracionamento em coluna cromatográfica de sílica gel utilizando $n$-hexano para obtenção dos hidrocarbonetos saturados (F1); $n$-hexano:diclorometano $(8: 2 \mathrm{v} / \mathrm{v})$ para os hidrocarbonetos aromáticos (F2) e diclorometano:metanol $(9: 1 \mathrm{v} / \mathrm{v})$ para os compostos polares (F3). Os hidrocarbonetos saturados interações polares. A adição de reagentes que possuem os prótons $\mathrm{H}^{+}$pode atender a essa exigência. $\mathrm{O}$ próton $\mathrm{H}^{+}$pode interagir eficazmente com as posições polares das moléculas de asfalteno e, desse modo, reduzir as interações polares. Portanto, a reação de degradação de asfalteno em meio ácido é preferida (Liao \& Geng 2002).

Baseado neste contexto e com o intuito de ampliar o conhecimento sobre o fenômeno natural de biodegradação em reservatórios de petróleo, o objetivo deste trabalho foi realizar um estudo comparativo dos biomarcadores presentes nas frações maltênica (maltenos livres no petróleo) e asfaltênica (maltenos ocluídos) das amostras de arenito asfáltico da Formação Piramboia, Bacia do Paraná, e enfatizar a importância geoquímica deste estudo levando em consideração a influência da biodegradação nos parâmetros avaliados. ou mesmo para caracterizar potenciais reservatórios. O óleo extraído dos arenitos asfálticos é caracterizado como pesado (aproximadamente $5^{\circ} \mathrm{API}$ ), "imaturo", de alta viscosidade e com médio a alto teor de enxofre ( 2 a $3 \%$ em peso), onde a maturação da matéria orgânica ocorreu principalmente devido à ação térmica das intrusões ígneas na Bacia do $\mathrm{Pa}$ raná, caracterizando-o como um sistema petrolífero atípico (Cerqueira \& Santos Neto 1986, Trigüis 1986, Thomaz Filho 2008). Análises geoquímicas por meio de biomarcadores revelam que a maior parte do óleo recuperado nesta Formação possui dominância de $n$-alcanos de elevado peso molecular, abundância de gamacerano e evidências de elevados níveis de biodegradação (Martins 2013).

foram analisados por cromatografia gasosa acoplada a detector de ionização em chama (CG-DIC) e por cromatografia gasosa acoplada a espectrometria de massas (CG-EM) para obtenção dos parâmetros geoquímicos. As condições de análise por CG-DIC foram: cromatógrafo Agilent $6890 \mathrm{~N}$, utilizando ar sintético, $\mathrm{H}_{2}$ e $\mathrm{N}_{2}$ como gases de chama, coluna capilar de sílica fundida HP-5 (30 m x 0,32 mm x 0,25 $\mu \mathrm{m})$ e programação linear de temperatura de $40^{\circ} \mathrm{C}$ a $320^{\circ} \mathrm{C}$ $\left(2,5^{\circ} \mathrm{C} / \mathrm{min}\right)$ por 18 minutos. A temperatura do injetor e do detector foi de 280 e $340^{\circ} \mathrm{C}$, respectivamente. Para o CG-EM utilizou-se cromatógrafo Agilent 6890N, acoplado a um analisador seletivo de massas Agilent 5973-MSD, operando com uma fonte de elétrons com energia de ionização de $70 \mathrm{eV}$, coluna capilar de sílica fundida do tipo DB-5MS (30 m x 0,25 mm x 0,25 mm) e programação linear de temperatura de $60^{\circ} \mathrm{C}$ por $2 \mathrm{~min}\left(22^{\circ} \mathrm{C} / \mathrm{min}\right)-200^{\circ} \mathrm{C}$ por $3 \mathrm{~min}\left(3^{\circ} \mathrm{C} / \mathrm{min}\right)-320^{\circ} \mathrm{C}$ por 25 minutos. A temperatura do injetor e da linha de transferência foi de $280^{\circ} \mathrm{C}$. Utilizou-se o método de monitoramento seletivo de íons (SIM - Selected Ions Monitoring) de acordo com cada classe de compostos avaliados. Foi injetado $1 \mu \mathrm{L}$ da amostra diluída em $n$-hexano na concentração de $0,02 \mathrm{mg} / \mu \mathrm{L}$. 


\section{RESULTADOS E DISCUSSÃO}

A análise SARA é comumente utilizada como um estudo preliminar dos componentes predominantes no petróleo. No entanto, não é possível, somente com esta análise, obter maior detalhamento da composição do petróleo, o que é feito com o uso de outros métodos de análise. Ainda assim, este conhecimento preliminar se faz necessário para diferenciar grupos de amostras e, por isso, foi utilizado neste trabalho com o intuito de visualizar as diferenças entre os maltenos livres e os ocluídos na estrutura asfaltênica. A diferença entre os maltenos livres (ML) e os ocluídos (MO) em termos de componentes SARA (saturados, aromáticos, resinas e asfaltenos) pode ser observada por meio do diagrama ternário da Figura 1.

Os maltenos livres apresentaram teor de resinas e asfaltenos $(64-72 \%)$ bem maior do que o teor de saturados (16-27\%) e de aromáticos (9-18\%), evidenciando que todas as amostras são biodegradadas, sendo que a amostra 12 foi a que apresentou maior teor de compostos polares $(72 \%)$. Por outro lado, observa-se que a fração ocluída pelo asfalteno das amostras 09, 12 e 25 apresentou um teor mais elevado de hidrocarbonetos saturados e aromáticos quando comparado à fração de malteno livre. Isto mostra que a estrutura asfaltênica preserva os $n$-alcanos da biodegradação. Segundo Liao e Geng (2002) isso ocorre porque a fração asfaltênica aprisiona e protege a fração maltênica, preservando assim os compostos saturados e aromáticos dos processos de alteração secundária. De forma análoga, Azevedo et al. (2009), em seu trabalho, concluíram que houve uma proteção dos óleos ocluídos pelas estruturas asfaltênicas, possibilitando assim uma análise geoquímica do óleo original. A amostra 26, ao contrário, apresentou um menor teor de hidrocarbonetos saturados quando comparado à fração livre. Isso pode ser atribuído à pouca quantidade de amostra utilizada na reação de oxidação, perdas durante o procedimento experimental ou, ainda, a baixa reatividade do asfalteno dessa amostra frente ao tratamento oxidativo.

Os percentuais de saturados e aromáticos para a fração ocluída em relação à massa inicial de asfaltenos precipitados para as quatro amostras foram de 0,$34 ; 0,09 ; 0,53 ; 0,19 \mathrm{e}$ 0,$32 ; 0,17 ; 0,56$ e $0,50 \%$ respectivamente. Os diferentes valores podem ser atribuídos às diferenças estruturais entre os asfaltenos dessas amostras, ou ainda que o asfalteno da amostra 25 (que apresentou maiores porcentagens, 0,53\% para HCs saturados e $0,56 \%$ para aromáticos) seja mais reativo face ao tratamento oxidativo.

Com o intuito de detalhar a composição do petróleo, um dos resultados mais significativos deste trabalho foi observado a partir da análise do íon-fragmento $\mathrm{m} / \mathrm{z} 85$ (Figura 2), onde foi possível observar a liberação dos hidrocarbonetos que estavam ocluídos na estrutura asfaltênica, em contraste com a ausência desses compostos nos maltenos livres.

Nos cromatogramas da Figura 2 observa-se, para todas as amostras de malteno livre, a ausência de $n$-alcanos de baixa a alta massa molecular e a sua preservação pela estrutura

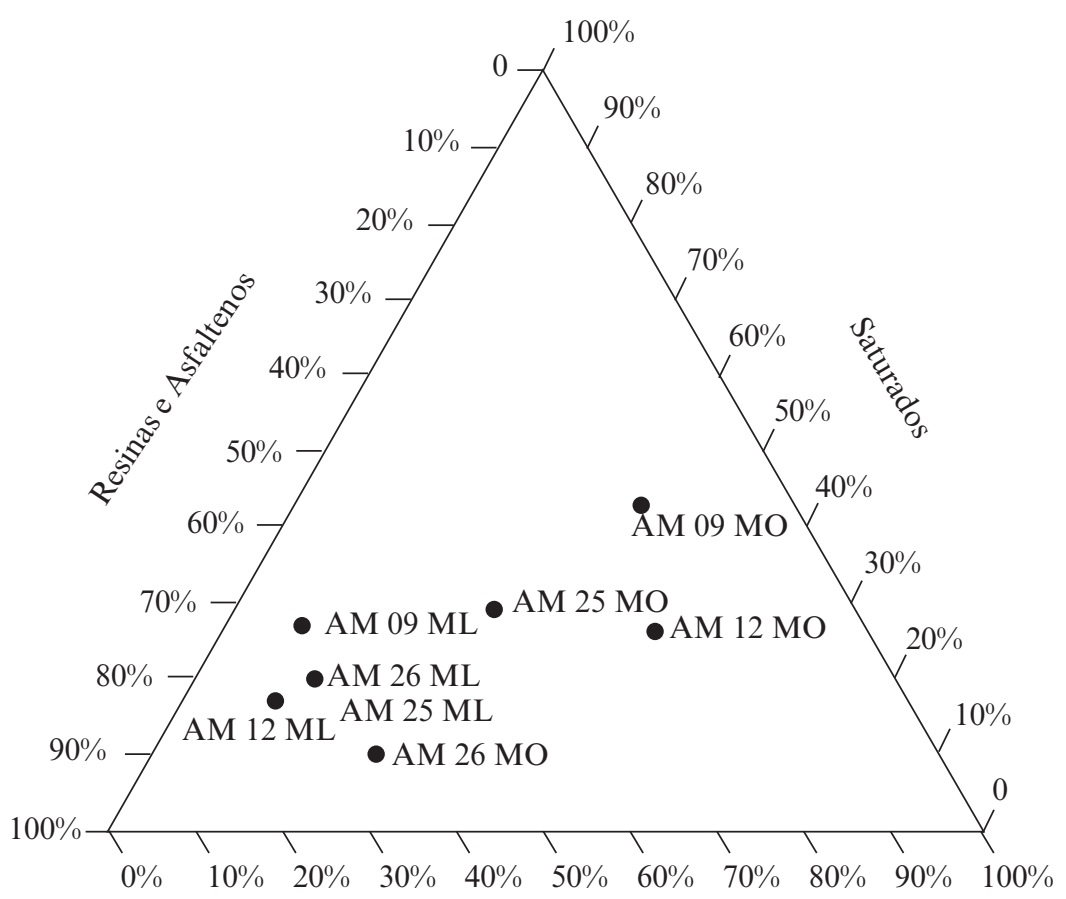

Aromáticos
Figura 1

Diagrama ternário das proporções de hidrocarbonetos saturados, aromáticos, resinas e asfaltenos para os maltenos livres e ocluídos. 
asfaltênica com uma distribuição unimodal, e ocorrência dos hidrocarbonetos de alta massa molecular nas amostras de malteno ocluído. A análise do perfil em $\mathrm{m} / z 85$ é mais uma evidência do eficiente fenômeno de proteção dos compostos ocluídos pela estrutura asfaltênica, comprovando a sua utilidade para obter informações sobre origem e paleoambiente deposicional do óleo original.

As Figuras 3 e 4 mostram uma comparação entre os cromatogramas para os íons-fragmentos $\mathrm{m} / \mathrm{z} 191$ (análise de terpanos) e $m / z 217$ (análise de esteranos), respectivamente, obtidos tanto para os maltenos livres quanto para os maltenos ocluídos.

A Tabela 1 apresenta os parâmetros geoquímicos avaliados para os maltenos livres e ocluídos.

Com o aumento da biodegradação, os valores das ra-

(A) AM 09

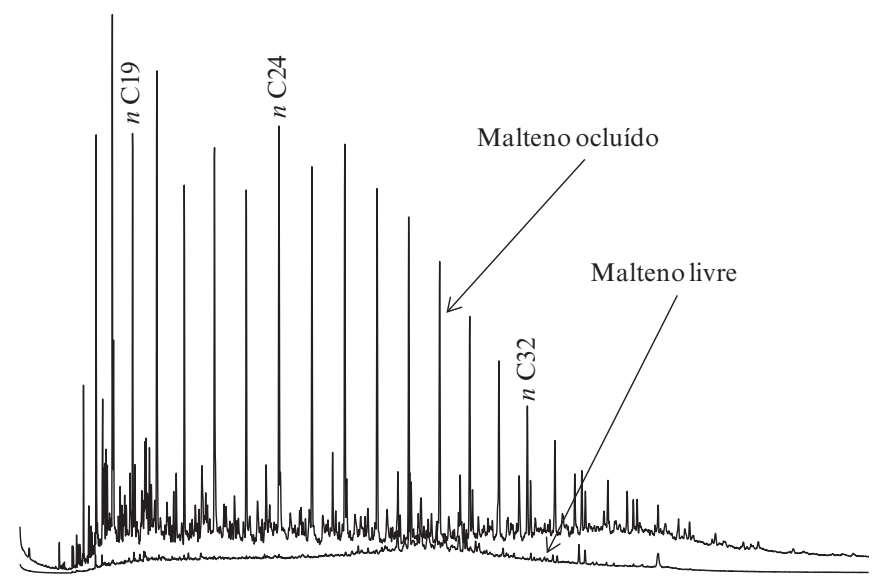

(C) AM 25

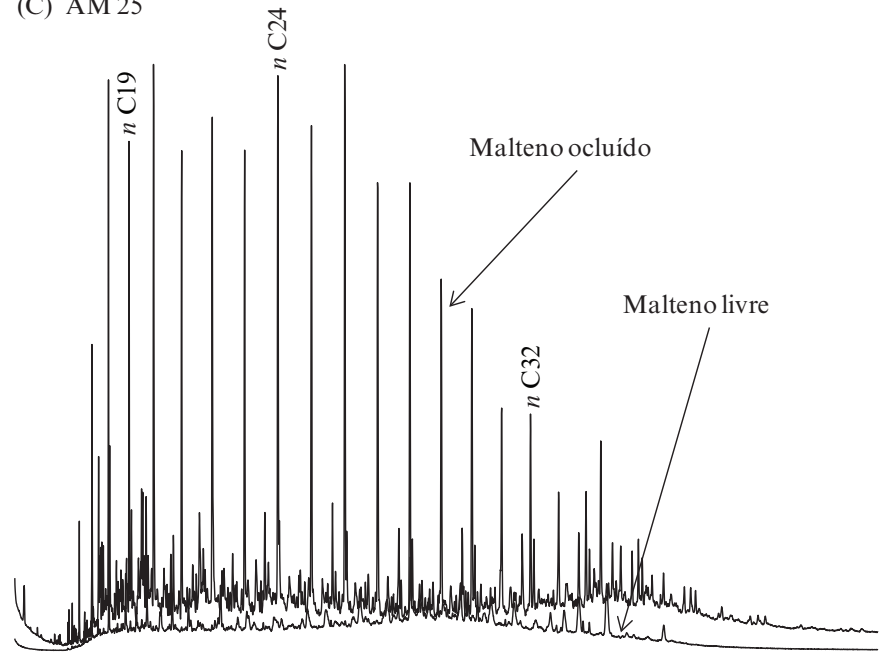

zões $\mathrm{Pr} / n$-C17 e F/n-C18 tendem a aumentar, uma vez que os $n$-alcanos são preferencialmente biodegradados. Nas amostras com biodegradação moderada (nível 3 na escala de Peters \& Moldowan, PM), as razões $\operatorname{Pr} / n$-C17 e F/n-C18 são mais elevadas em relação aos óleos não biodegradados ou com biodegradação leve (PM 2). Em contrapartida, em óleos severamente biodegradados $(\mathrm{PM}>6)$, essas razões tendem a zero devido à ausência destes compostos, observando-se apenas uma elevação na linha base (aumento da UCM). A razão $\operatorname{Pr} / F$ normalmente é utilizada para avaliar o ambiente deposicional e a matéria orgânica que deu origem a este óleo (Peters et al. 2005, Marynowski et al. 2007). Neste trabalho, observou-se a presença de pristano $(\operatorname{Pr})$ e fitano (F) com predominância de Pr sobre F nas amostras 09 e 26 (razão das áreas dos picos $\operatorname{Pr} / \mathrm{F}>1$, Tabela 1) nos maltenos

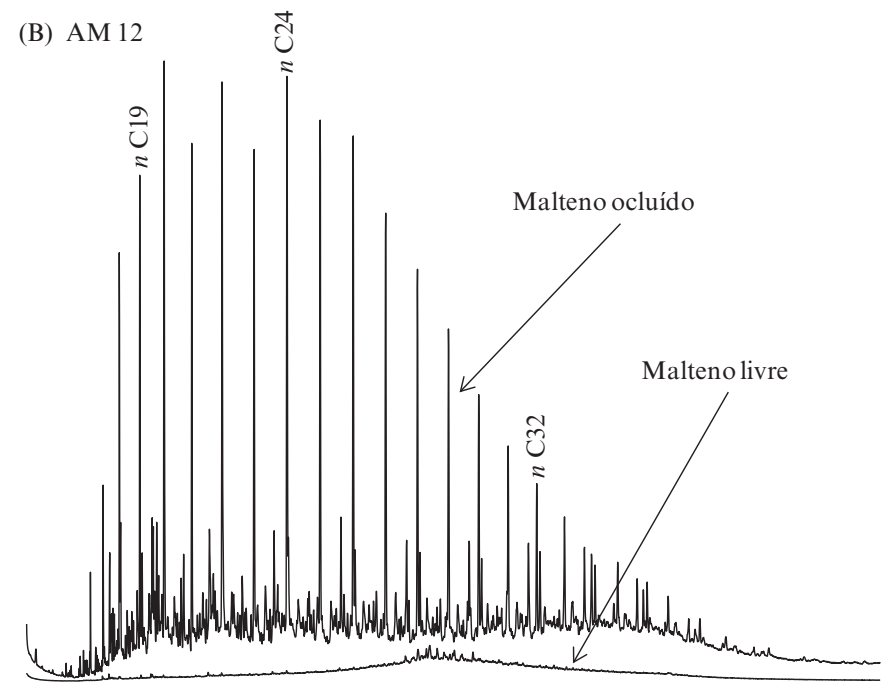

(D) AM 26

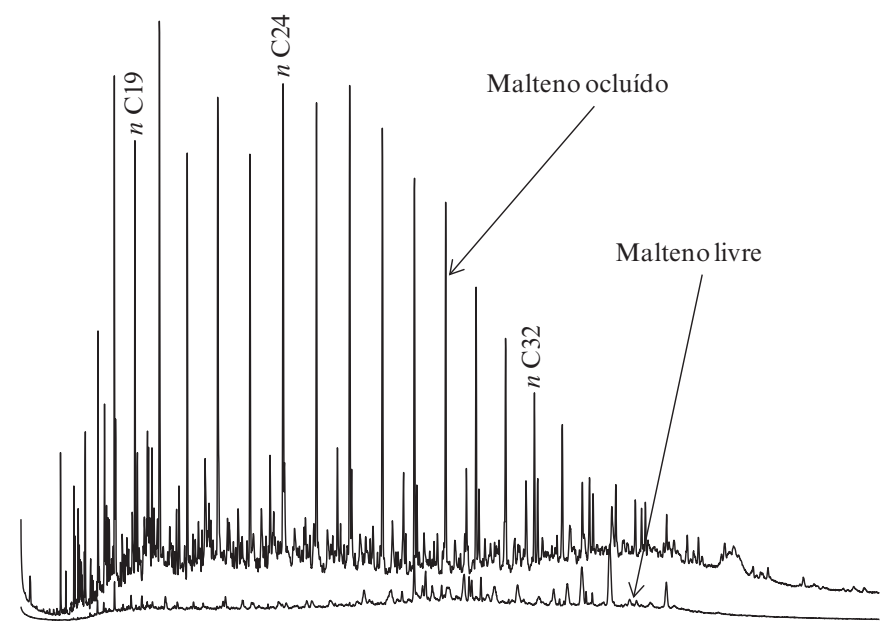

Figura 2

Comparação entre maltenos livres e ocluídos utilizando monitoramento do íon-fragmento $\mathrm{m} / \mathrm{z} 85$. 
AM 09 Malteno livre

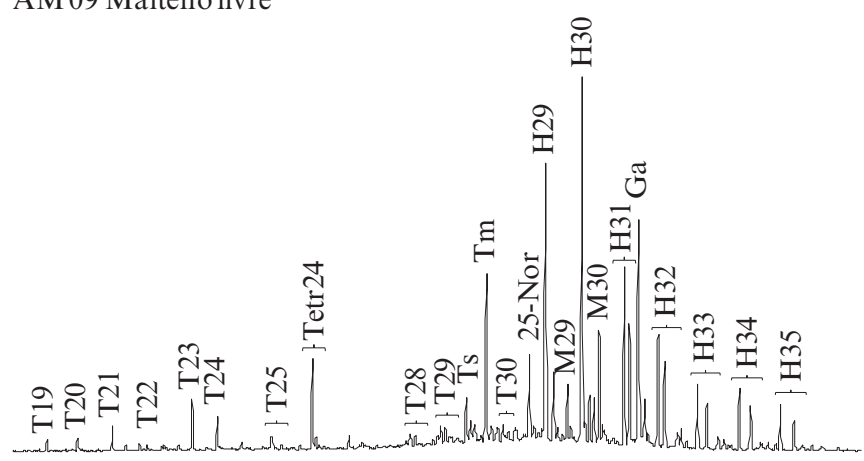

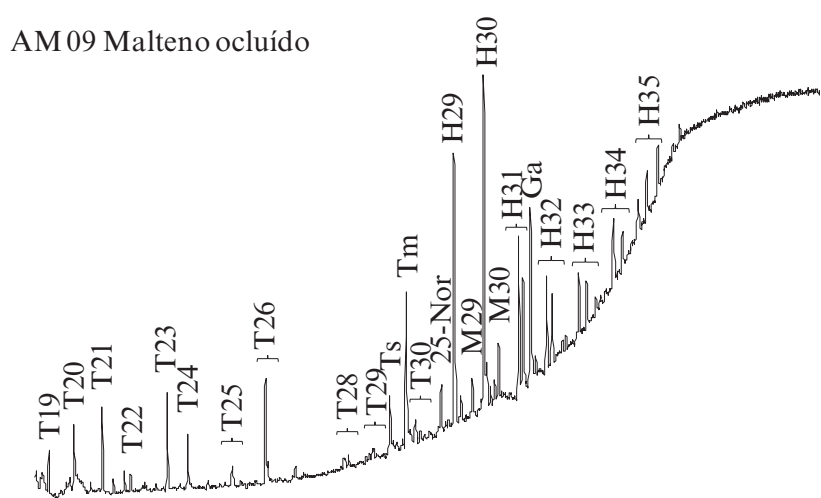

AM 12 Malteno livre
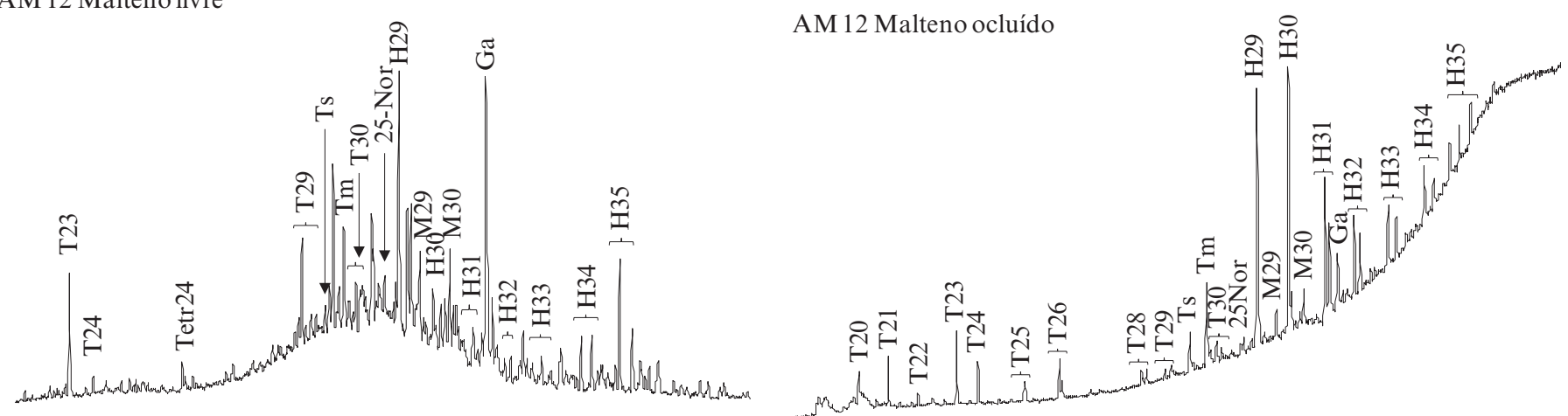

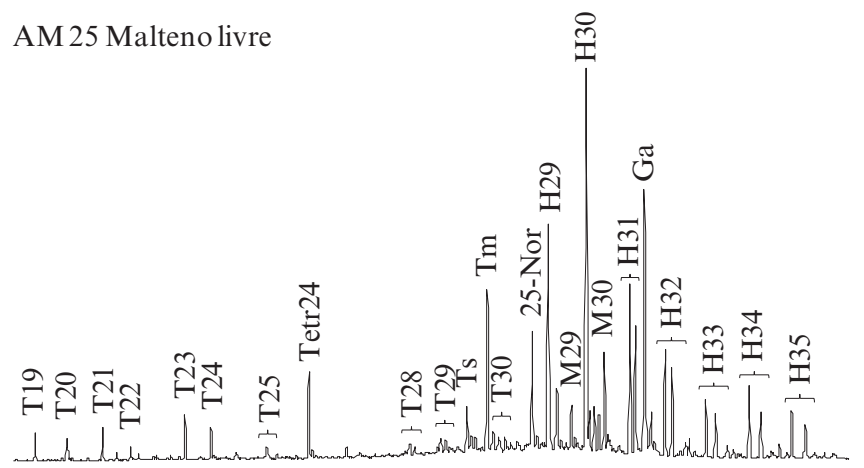

AM 25 Malteno ocluído
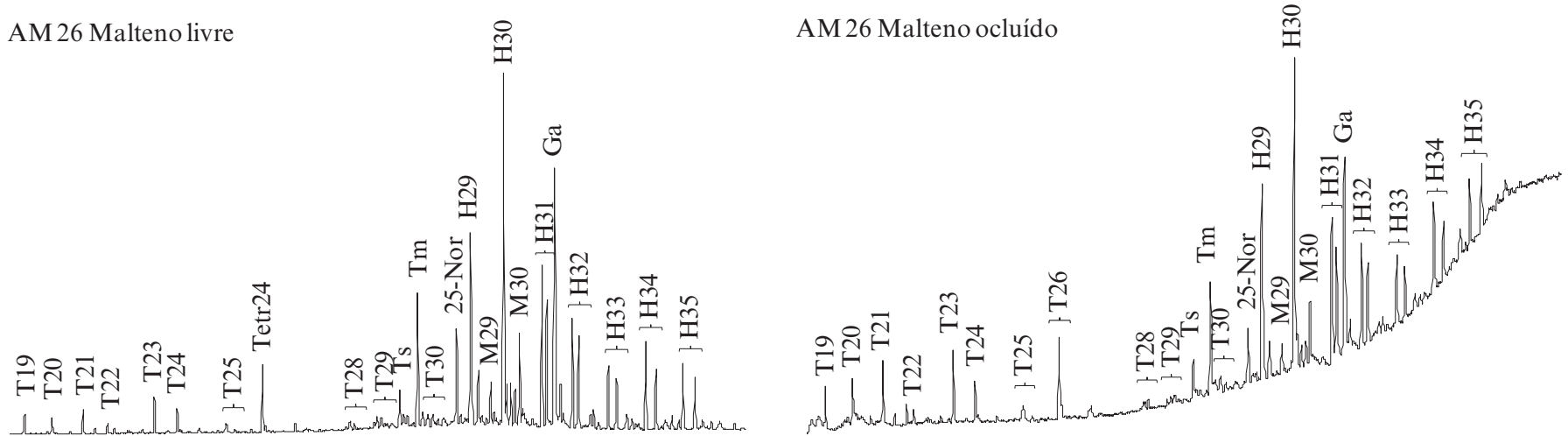

Figura 3

Comparação entre os cromatogramas para o íon-fragmento $m / z 191$ para os maltenos livres e ocluídos. 
AM 09 Malteno livre

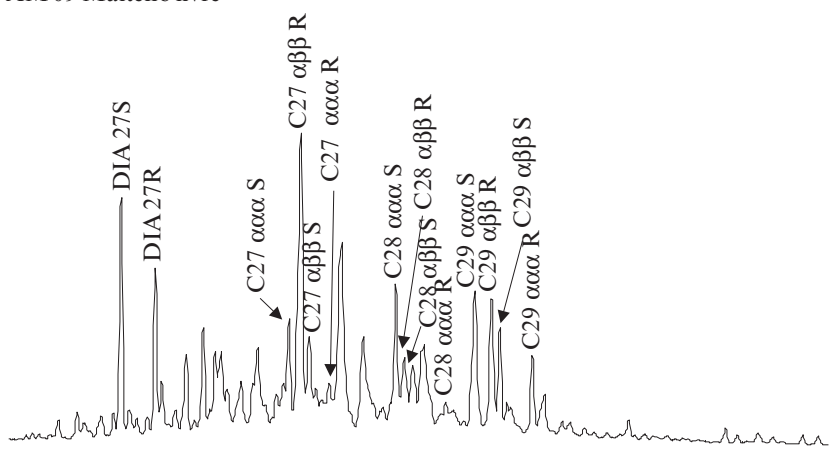

AM09 Malteno ocluído
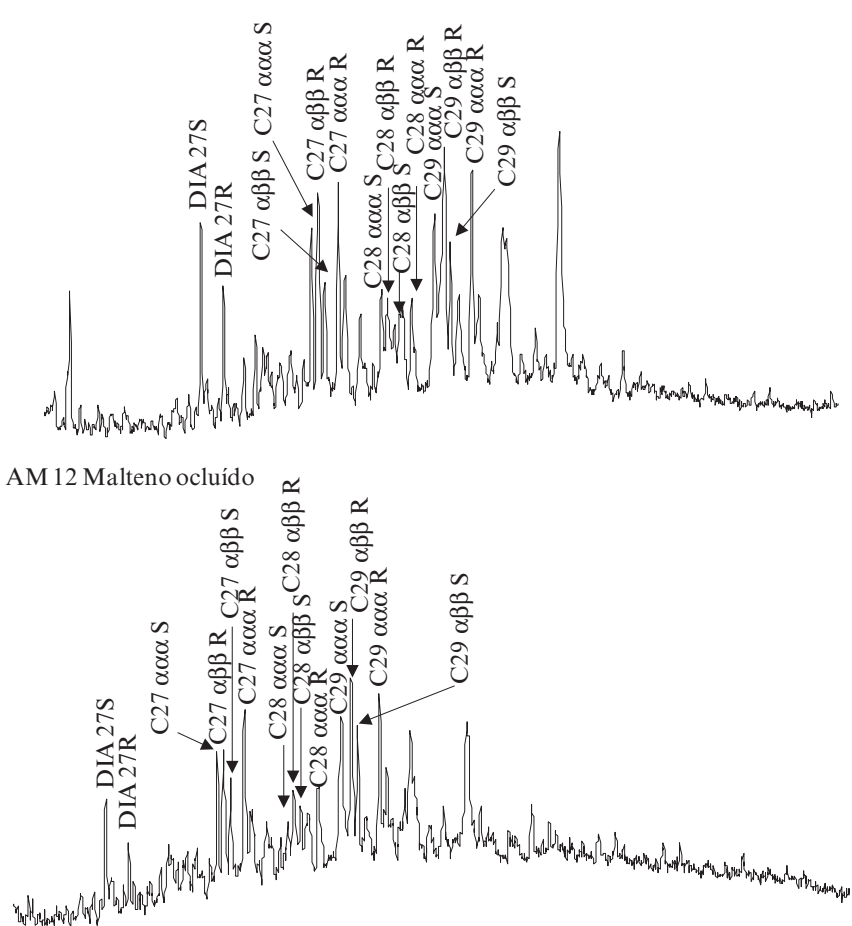

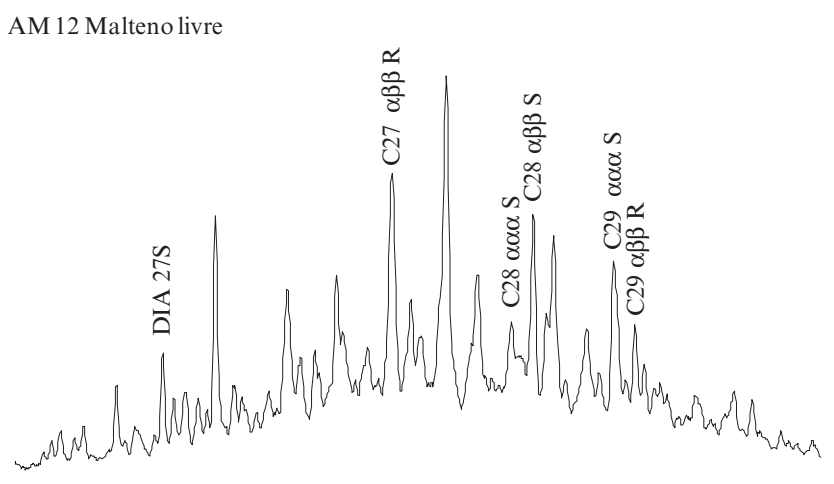

AM 25 Malteno livre
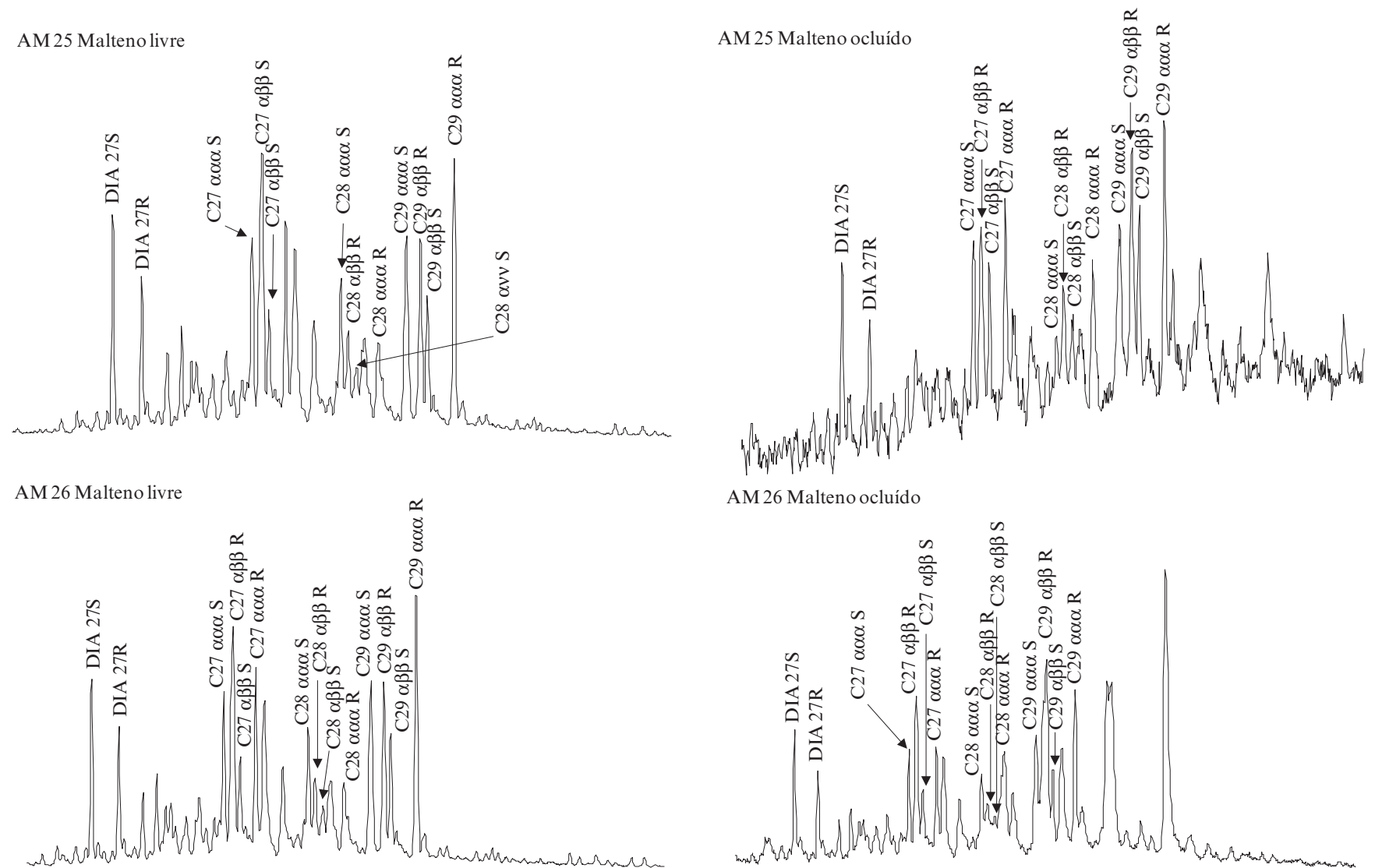

Figura 4

Comparação entre os cromatogramas

para o íon-fragmento $\mathrm{m} / \mathrm{z} 217$ para os maltenos livres e ocluídos. 


\begin{tabular}{|c|c|c|c|c|c|c|c|c|}
\hline \multirow{3}{*}{$\begin{array}{l}\text { Parâmetros } \\
\text { geoquímicos }\end{array}$} & \multicolumn{8}{|c|}{ Amostras } \\
\hline & \multicolumn{4}{|c|}{ Malteno livre } & \multicolumn{4}{|c|}{ Malteno ocluído } \\
\hline & AM 09 & AM 12 & AM 25 & AM 26 & AM 09 & AM 12 & AM 25 & AM 26 \\
\hline $\mathrm{Pr} / \mathrm{F}$ & 1,48 & $\mathrm{nd}^{*}$ & 0,78 & 1,04 & 0,93 & 0,63 & 0,52 & 0,55 \\
\hline $\mathrm{Pr} / \mathrm{n}-\mathrm{C} 17$ & 0,69 & nd & 3,81 & 1,29 & 0,46 & 1,16 & 0,44 & 0,86 \\
\hline $\mathrm{F} / \mathrm{n}-\mathrm{C} 18$ & 0,33 & nd & 13,47 & 1,27 & 0,43 & 0,40 & 0,45 & 0,45 \\
\hline $\mathrm{H} 29 / \mathrm{H} 30$ & 0,86 & 7,41 & 0,69 & 0,65 & 1,03 & 1,26 & 1,27 & 0,87 \\
\hline Tric/ Hop & 0,13 & 0,33 & 0,13 & 0,11 & 0,22 & 0,20 & 0,24 & 0,17 \\
\hline $\mathrm{H} 32 \mathrm{~S} /(\mathrm{S}+\mathrm{R})$ & 0,58 & 0,41 & 0,56 & 0,56 & 0,61 & 0,59 & 0,55 & 0,56 \\
\hline $\mathrm{Ga} / \mathrm{H} 30$ & 0,66 & 10,25 & 0,80 & 0,87 & 0,69 & 0,32 & 0,30 & 0,87 \\
\hline 25-Nor/H30 (191) & 0,21 & 0,78 & 0,28 & 0,27 & 0,15 & 0,06 & 0,03 & 0,18 \\
\hline $\mathrm{S} /(\mathrm{S}+\mathrm{R})(\mathrm{C} 29 \alpha \alpha \alpha)$ & 0,73 & nd & 0,48 & 0,47 & 0,49 & 0,59 & 0,47 & 0,41 \\
\hline$\beta \beta /(\alpha \alpha+\beta \beta)(C 29)$ & 0,50 & 0,25 & 0,46 & 0,57 & 0,44 & 0,44 & 0,34 & 0,34 \\
\hline $\mathrm{M} 30 / \mathrm{H} 30$ & 0,27 & 2,49 & 0,24 & 0,25 & 0,17 & 0,12 & 0,13 & 0,20 \\
\hline DIA/REG & 0,29 & 0,10 & 0,16 & 0,15 & 0,16 & 0,10 & 0,13 & 0,15 \\
\hline
\end{tabular}

Tabela 1

Parâmetros geoquímicos calculados para os maltenos livres e ocluídos.

nd: não determinado

Pr/F: Pristano/Fitano; Pr/n-C17: Pristano/n-alcano C17; F/n-C18: Fitano/n-alcano C18; H29/ H30: $17 \alpha(H), 21 \beta(H)-C 30$-norhopano/17 $\alpha(H), 21 \beta(H)$-hopano $(\mathrm{m} / \mathrm{z} 191)$; Tric $/ \mathrm{Hop}=(\operatorname{Tr} 20+$ $\operatorname{Tr} 21+\operatorname{Tr} 22+\operatorname{Tr} 23+\operatorname{Tr} 24+\operatorname{Tr} 25 \mathrm{~S}+\operatorname{Tr} 25 \mathrm{R}+\operatorname{Tr} 28 \mathrm{~S}+\operatorname{Tr} 28 \mathrm{R}+\operatorname{Tr} 29 \mathrm{~S}+\operatorname{Tr} 29 \mathrm{R}+\operatorname{Tr} 30 \mathrm{~S}+\operatorname{Tr} 30 \mathrm{R})$ $(\mathrm{Ts}+\mathrm{Tm}+\mathrm{H} 29+\mathrm{H} 30+\mathrm{H} 31 \mathrm{~S}+\mathrm{H} 31 \mathrm{R}+\mathrm{H} 32 \mathrm{~S}+\mathrm{H} 32 \mathrm{R}+\mathrm{H} 33 \mathrm{~S}+\mathrm{H} 33 \mathrm{R}+\mathrm{H} 34 \mathrm{~S}+\mathrm{H} 34 \mathrm{R}+\mathrm{H} 35 \mathrm{~S}$ $+\mathrm{H} 35 \mathrm{R})(\mathrm{m} / \mathrm{z} 191) ; \mathrm{H} 32 \mathrm{~S} /(\mathrm{S}+\mathrm{R})=17 \alpha(\mathrm{H}), 21 \beta(\mathrm{H})$-bishomohopano $(22 \mathrm{~S}) / 17 \alpha(\mathrm{H}), 21 \beta(\mathrm{H})-$ bishomohopano $(22 \mathrm{~S}+22 \mathrm{R})(\mathrm{m} / \mathrm{z} 191) ; \mathrm{Ga} / \mathrm{H} 30=$ gamacerano/17 $\alpha(\mathrm{H}), 21 \beta(\mathrm{H})$-hopano $(\mathrm{m} / \mathrm{z}$ 191); 25 -Nor/H30 = 17 $\alpha(\mathrm{H}), 21 \beta(\mathrm{H})$-25-norhopano/17 $\alpha(\mathrm{H}), 21 \beta(\mathrm{H})$-hopano $(\mathrm{m} / \mathrm{z} 191) ; \mathrm{S}(\mathrm{S}+\mathrm{R})$ $(\mathrm{C} 29 \alpha \alpha \alpha)=\mathrm{C} 29 \alpha \alpha \alpha \mathrm{S} /(\mathrm{C} 29 \alpha \alpha \alpha \mathrm{S}+\mathrm{C} 29 \alpha \alpha \alpha \mathrm{R})(\mathrm{m} / \mathrm{z} 217) ; \beta \beta /(\alpha \alpha+\beta \beta)(\mathrm{C} 29)=(\mathrm{C} 29 \alpha \beta \beta S+$ $\mathrm{C} 29 \alpha \beta \beta \mathrm{R}) /(\mathrm{C} 29 \alpha \alpha \alpha \mathrm{S}+\mathrm{C} 29 \alpha \beta \beta \mathrm{R}+\mathrm{C} 29 \alpha \beta \beta \mathrm{S}+\mathrm{C} 29 \alpha \alpha \alpha \mathrm{R})(\mathrm{m} / \mathrm{z} 217) ; \mathrm{M} 30 / \mathrm{H} 30=17 \beta(\mathrm{H}), 21 \alpha(\mathrm{H})-$ moretano/17 $\alpha(\mathrm{H}), 21 \beta(\mathrm{H})$-hopano $(\mathrm{m} / \mathrm{z} 191)$; DIA/REG = (DIA27S + DIA27R) $/(\mathrm{C} 27 \alpha \alpha \alpha \mathrm{S}+$ $\mathrm{C} 27 \alpha \beta \beta R+C 27 \alpha \beta \beta S+C 27 \alpha \alpha \alpha R+C 28 \alpha \alpha \alpha S+C 28 \alpha \beta \beta R+C 28 \alpha \beta \beta S+C 28 \alpha \alpha \alpha R+C 29 \alpha \alpha \alpha S+$ $C 29 \alpha \beta \beta R+C 29 \alpha \beta \beta S+C 29 \alpha \alpha \alpha R)(m / z 217)$.

livres. Na amostra 25 o fitano é o isoprenoide predominante, e na amostra 12 observa-se ausência de ambos isoprenoides. Por outro lado, nos maltenos ocluídos observou-se predominância de $\mathrm{F}$ sobre $\operatorname{Pr}$ (razão das áreas dos picos $\operatorname{Pr} / \mathrm{F}<1$, Tabela 1) para todas as amostras. Isto mostra que a razão $\mathrm{Pr} / \mathrm{F}$ deve ser usada com cautela para monitorar biodegradação de óleos em reservatório (Sun et al. 2005).

Baseando-se somente nas razões $\mathrm{Pr} / n-\mathrm{C} 17$ e $\mathrm{F} / n-\mathrm{C} 18$, observa-se que a amostra 09 para os maltenos livres possui os valores mais baixos, indicando que os $n$-alcanos estão em maior abundância relativa comparado aos isoprenoides, e que esta é a amostra menos biodegradada. Por outro lado, a amostra 12 possui o maior nível de biodegradação, visto que foi observada a ausência tanto dos $n$-alcanos quanto dos isoprenoides e, por isso, as razões não puderam ser calculadas. Nas amostras 25 e 26 os valores das razões estão relativamente altos, indicando que os isoprenoides estão em maior quantidade do que os $n$-alcanos e, comparadas com as demais, tais amostras encontram-se moderadamente biodegradadas, sendo que a amostra 25 apresenta um maior nível de biodegradação do que a amostra 26 .

De maneira geral, todos os cromatogramas em $\mathrm{m} / \mathrm{z}$ 191 obtidos para o malteno ocluído apresentaram o perfil característico de um óleo não biodegradado. A distribuição dos compostos $\mathrm{H} 29$ e H30 se mantém quase na mesma proporção entre os maltenos livres para as amostras 09, 25 e 26. Em contrapartida, ocorre um aumento significativo da razão H29/H30 para a amostra 12 (Tabela 1), sugerindo que o H29 é mais resistente à biodegradação que o H30 em amostras severamente biodegradadas $(\mathrm{PM}>6)$. Segundo Peters et al. (2005), esta razão também pode ser avaliada com relação à maturação, pois o norhopano (H29) é mais estável que hopano (H30) em altos níveis de maturidade termal. Assim, sugere-se que os maiores valores da razão H29/H30 para as amostras 09, 12 e 25 nos maltenos ocluídos, as quais estão mais protegidas da biodegradação, se deve à maior maturidade térmica quando comparada com a amostra $26(\mathrm{H} 29 / \mathrm{H} 30<1)$.

Ainda de acordo com a Figura 3, observa-se que há um aumento significativo do gamacerano entre os maltenos livres. Isso se deve ao fato de que quanto maior a biodegradação maior será a abundância do gamacerano $(\mathrm{Ga})$, visto que este apresenta uma maior resistência à degradação microbiana quando comparado ao $17 \alpha(\mathrm{H}), 21 \beta(\mathrm{H})$-hopano (H30). Isto faz com que a razão $\mathrm{Ga} / \mathrm{H} 30$ seja maior nas amostras de malteno livre, principalmente na amostra 12 $(\mathrm{Ga} / \mathrm{H} 30=10,25$, Tabela 1$)$, quando comparada às amostras de maltenos ocluídos, os quais estão mais protegidos da biodegradação, fazendo com que a razão Ga/H30 seja menor (Tabela 1).

Com relação aos terpanos tricíclicos, para as amostras 09, 25 e 26 o perfil de distribuição das frações livre e ocluída é similar, estando apenas alguns compostos com maior abundância para o malteno ocluído. O mesmo não ocorre para a 
amostra 12, onde a maior parte dos terpanos tricíclicos do malteno livre foi consumida pela biodegradação. No trabalho realizado por Liao e Geng (2002) na Bacia de Songliao (China), observou- se que os terpanos tricíclicos de C20 até C26 estavam ausentes para as amostras de malteno livre, mas estes mesmos terpanos estavam presentes nas amostras de malteno ocluído, e ainda apresentaram maior abundância relativa do que a dos terpanos pentacíclicos. Para Azevedo et al. (2009), entretanto, os compostos terpanos tricíclicos de C20 a C26 foram obsevados tanto para o malteno livre como para o ocluído, notando-se ainda que os terpanos tricíclicos estavam em maior quantidade do que os terpanos pentacíclicos nos dois casos. Neste trabalho também se observou a presença desses compostos tanto para o malteno livre quanto para o malteno ocluído, com exceção da amostra 12 livre, na qual alguns terpanos tricíclicos estão ausentes.

Outro resultado observado foi o aumento do composto 25 -norhopano em todas as amostras de maltenos livres. Isso se deve ao fato de que com o aumento da biodegradação mais compostos são liberados. De acordo com Peters et al. (2005), existem três hipóteses para a origem dos 25-norhopanos em reservatório: i) alguns micro-organismos produzem 25-norhopanos, hopanos e seus precursores biológicos. Neste caso, a biodegradação severa remove os hopanos com consequente enriquecimento de 25-norhopanos; ii) os 25-norhopanos são originados por meio da remoção da metila em C-10 dos hopanos devido ao ataque microbiano; e iii) existem diferentes grupos de micro-organismos no reservatório. Aqueles que estão presentes na formação da matéria orgânica sedimentar não produzem 25 -norhopanos ou seu precursor biológico. E aqueles que são efetivamente responsáveis pela biodegradação de petróleo produzem 25-norhopanos e não degradam hopanos.

Analisando a Figura 3 e a Tabela 1 notou-se que, mesmo em menor abundância que os hopanos (razão 25 -Nor/H30<1), os 25 -norhopanos estão presentes em todas as amostras da fração ocluída. Como os compostos desta fração estão protegidos da biodegradação, sugere-se que os 25-norhopanos possam ser provenientes de alguns micro-organismos durante o processo diagenético conforme relatado por Peters et al. (2005) na primeira hipótese para a origem desses compostos.

Os perfis de distribuição de esteranos $(m / z$ 217) nas frações de biomarcadores livres e ocluídos, apresentados na Figura 4, também mostram a presença de evidências dos efeitos de biodegradação para a fração de biomarcadores livres e a preservação nos maltenos ocluídos. Vale ressaltar que a liberação dos esteranos da estrutura asfaltênica, utilizando oxidação branda, foi mais eficiente que a dos hopanos.

A preferência da biodegradação dos esteranos normalmente segue a ordem: $\alpha \alpha \alpha 20 R>>\alpha \beta \beta 20 R+20 S{ }^{3} \alpha \alpha \alpha$ $20 S>>$ diasteranos e geralmente diminui com o aumento do número de carbonos $(\mathrm{C} 27>\mathrm{C} 28>\mathrm{C} 29>\mathrm{C} 30)$ (Seifert \& Moldowan 1979, Chosson et al. 1991). Isto já foi obser- vado tanto em reservatórios (Dajiang et al. 1988) quanto em laboratório (Goodwin et al. 1981, Chosson et al. 1991, Da Cruz 2009, Da Cruz et al. 2011). C27-C30 $\alpha \alpha \alpha$ 20R é a configuração biológica dos esteranos e, por isso, eles são mais rapidamente consumidos ou biodegradados do que os outros isômeros. Outra observação importante é que a suscetibilidade relativa de $\alpha \beta \beta 20 R, \alpha \beta \beta 20 S$ e $\alpha \alpha \alpha 20 S$ varia consideravelmente e depende do tipo de micro-organismo envolvido e das condições do reservatório e/ou da simulação em laboratório (Peters et al. 2005, Da Cruz 2009, Da Cruz et al. 2011) e, assim, pode ocorrer remoção destes compostos ao mesmo tempo ou os isômeros $\alpha \beta \beta 20 R$ e $20 S$ podem ser removidos antes ou depois do $\alpha \alpha \alpha 20 S$ (Seifert \& Moldowan 1979).

A importância de entender a preferência de biodegradação dos esteranos reside no fato de que a isomerização dos esteranos C29 com relação aos isômeros $\alpha \alpha \alpha(20 R+20 S)$ e $\alpha \beta \beta(20 R+20 S)$ e razão diasteranos (DIA)/esteranos regulares (REG), são os parâmetros geoquímicos mais utilizados para classificar o grau de maturação de óleos e a remoção seletiva dos epímeros devido à biodegradação afeta os valores destas razões (Peters et al. 2005). Neste estudo não foi possível estabelecer uma ordem preferencial de biodegradação para os esteranos regulares, como já demonstrado por outros autores (Seifert \& Moldowan 1979, Chosson et al. 1991, Peters et al. 2005). No entanto, em geral, observou-se uma preservação dos esteranos com configuração biológica $(\alpha \alpha \alpha 20 R)$ nos maltenos ocluídos, quando comparado aos livres, especialmente para a amostra 12 , que apresenta predominância dos isômeros de configuração geológica $\alpha \beta \beta$ nos maltenos livres. As amostras 25 e 26 apresentaram perfis semelhantes para maltenos livres e ocluídos, evidenciando biodegradação menos severa quando comparado às demais amostras.

Os valores obtidos para as razões $S /(S+R)(C 29 \alpha \alpha \alpha), \beta \beta /$ $(\alpha \alpha+\beta \beta)(C 29)$ e DIA/REG (Tabela 1$)$, utilizadas para avaliar a maturidade térmica, em geral, foram menores nos maltenos ocluídos quando comparado aos livres. Isto pode ser atribuído à maior maturidade térmica dos maltenos livres em relação aos ocluídos, os quais, uma vez ocluídos pela estrutura asfaltênica, ficam protegidos tanto dos processos de alteração secundária quanto do efeito termocatalítico responsável pelas reações de isomerização (Azevedo et al. 2009).

A análise comparativa dos biomarcadores revelou que os maltenos livres são severamente biodegradados $(\mathrm{PM}>6)$ e, ainda, mostrou a eficiência da reação de oxidação branda para a liberação dos compostos ocluídos. Assim, destaca-se que a importância geoquímica do estudo de compostos ocluídos em estruturas asfaltênicas refere-se principalmente ao fato desses componentes não estarem mascarados pela biodegradação, tornando assim o estudo dos parâmetros mais confiável. Mesmo a fração ocluída sendo menos evoluída termicamente do que a livre, esta análise é eficaz, devido ao malteno obtido ser remanescente do óleo original. 


\section{CONCLUSÃO}

O estudo dos biomarcadores liberados da estrutura asfaltênica das amostras biodegradadas, em comparação com os compostos livres, permitiu concluir que em todas as amostras de malteno ocluído os componentes do petróleo foram protegidos pela estrutura asfaltênica. Já nos óleos livres, estes compostos se encontravam biodegradados em sua quase totalidade. Isto foi observado por meio da análise SARA e pelo monitoramento seletivo dos íons-fragmentos em $m / z$ 85, 177, 191 e 217 (por CG-EM).

\section{AGRADECIMENTOS}

Os autores agradecem o auxílio financeiro do Programa de Formaação de Recursos Humanos da Agência Nacional do Petróleo (PRH20/ANP).

\section{REFERÊNCIAS}

Azevedo D. de A., da Silva T.F., da Silva D.B. 2009. Avaliação geoquímica de biomarcadores ocluídos em estruturas asfaltênicas. Química Nova, 32:1770-1776.

Badre S., Goncalves C.C., Norinaga K., Gustavson G., Mullins O.C. 2006. Molecular size and weight of asphaltene and asphaltene solubility fractions from coals, crude oils and bitumen. Fuel, 85:1-11.

Behar F., Pelet R., Roucache J. 1984. Geochemistry of asphaltenes. Organic Geochemistry, 6:587-595.

Blanc P. \& Connan J. 1994. Preservation, degradation, and destruction of trapped oil. Memoirs-American Association of Petroleum Geologists, 60:237-247.

Calemma V., Rausa R., D’Anton P., Montanari L. 1998. Characterization of asphaltenes molecular structure. Energy \& Fuels, 12:422-428.

Cerqueira J.R. \& Santos Neto E.V. 1986. Papel das intrusões de diabásio no processo de geração de hidrocarbonetos na Bacia do Paraná. In: $3^{\circ}$ Congresso Brasileiro de Petróleo. Óleo e gás: Cruzando novas fronteiras, TT-73, p. 1-15, Rio de Janeiro.

Chosson P., Connan J., Dessort D., Lanau C. 1992. In vitro biodegradation of steranes and terpanes: A clue to understanding geological situations. In: Biological markers in sediments and petroleum. Moldowan J.M., Albrecht P., Philp R.P. (eds.) Prentice-Hall, Englewood Cliffs, p. 320-349.

Chosson P., Lanau C., Connan J., Dessort D. 1991. Biodegradation of refractory hydrocarbon biomarkers from petroleum under laboratory conditions. Nature, 351:640-642.

Connan J. 1984. Biodegradation of crude oils in reservoirs. Advances in petroleum geochemistry, 1:299-335.

Da Silva A.A. 2008. Estudos geoquímicos com evidências paleoambientais, maturação e biodegradação dos óleos de diferentes origens da Bacia Potiguar, Brasil. Síntese de biomarcadores de esterois. Tese de doutorado, Instituto de Química, Universidade Estadual de Campinas, 411 p.

Da Cruz G.F., Dos Santos N.E.V., Marsaioli A.J. 2008. Petroleum degradation by aerobic microbiota from the Pampo Sul oil field, Campos Basin, Brazil. Organic Geochemistry, 8:1204-1209.

Da Cruz G.F. 2009. Biodegradação aeróbia e anaeróbia de petróleo do Campo Pampo Sul, Bacia de Campos, RJ. Tese de Doutorado), Instituto de Química, Universidade Estadual de Campinas, 345 p.

Da Cruz G.F., Vasconcellos S.P., Angolini C.F., Dellagnezze B.M., Garcia I.N., Oliveira V.M., Dos Santos N.E.V., Marsaioli A.J. 2011. Could petroleum 
biodegradation be a joint achievement of aerobic and anaerobic microrganisms in deep sea reservoirs? AMB Express, 1:1-10.

Da Cruz G.F. \& Marsaioli A.J. 2012. Processos naturais de biodegradação do petróleo em reservatórios, Química Nova, 35:1628-1634.

Dajiang Z., Difan H., Jinchao L. 1988. Biodegraded sequence of Karamay oils and semi-quantitative estimation of their biodegraded degrees in Junggar Basin, China. Organic Geochemistry, 1:295-302.

De Lima S.G., Steffen R.A., Reis F. de A.M., Koike L., Neto E.V.S., Cerqueira J.R., Lopes J.A. 2010. Propyl ergostanoic acids: Possible new indicator for oil biodegradation. Organic Geochemistry, 4:325-339.

Ekweozor C.M. 1986. Characterisation of the non-asphaltene products of mild chemical degradation of asphaltenes. Organic Geochemistry, 10:1053-1058.

Ekweozor C.M. 1984. Tricyclic terpenoid derivatives from chemical degradation reactions of asphaltenes. Organic Geochemistry, 6:51-61.

Garcia M. de A., Ribeiro H.J.P.S., de Souza E.S., Triguis J.A. 2011. Correlação entre a faciologia e a geoquímica orgânica dos tar sands da Formação Piramboia, Triássico da Bacia do Paraná, Fazenda Betumita, no estado de São Paulo. Geociências (São Paulo), 30:357-369.

Gawrys K.L. \& Kilpatrick P.K. 2005. Asphaltenic aggregates are polydisperse oblate cylinders. Journal of colloid and interface science, 288:325-334.

Goodwin N., Park P., Rawlinson A. 1981. Crude oil biodegradation under simulated and natural conditions. In: Bjorøy M., Albrecht C., Cornford C., de Groot k., Eglington G., Galimov E., Leythaeuser D., Pelet R., Rullkotter J., Speers G. (eds.) Advances in organic geochemistry, New York, J. Wiley \& Sons, p. 650-658.

Gürgey K. 1998. Geochemical effects of asphaltene separation procedures: Changes in sterane, terpane, and methylalkane distributions in maltenes and asphaltene co-precipitates. Organic geochemistry, 29:1139-1147.

Hunt J.M. 1996. Petroleum geology and geochemistry. Freeman and Company, New York, $743 \mathrm{p}$.

Larter S., Huang H., Adams J., Bennett B., Jokanola O., Oldenburg T., Jones M., Head I., Riediger C., Fowler M. 2006. The controls on the composition of biodegraded oils in the deep subsurface: Part II - geological controls on subsurface biodegradation fluxes and constraints on reservoir-fluid property prediction, AAPG Bulletin, American Association Of Petroleum Geologists, 90:921-938.

Liao Z. \& Geng A. 2002. Characterization of $n$-C7 soluble fractions of the products from mild oxidation of asphaltenes. Organic Geochemistry, 33:14771486.

Liao Z., Geng A., Graciaa A., Creux P., Chrostowska A., Zhang Y. 2006a. Saturated hydrocarbons occluded inside asphaltene structures and their geochemical significance, as exemplified by two Venezuelan oils. Organic Geochemistry, 37:291-303.

Liao Z., Graciaa A., Geng A., Chrostowska A., Creux P. 2006b. A new lowinterference characterization method for hydrocarbons occluded inside asphaltene structures. Applied geochemistry, 21:833-838.

Mackenzie A.S. 1984. Applications of biological markers in petroleum geochemistry. Advances in petroleum geochemistry, 1:1-210.

Mansoori G.A. 1997. Modeling of asphaltene and other heavy organic depositions. Journal of Petroleum Science and Engineering, 17:101-111.

Martins L.L. 2013. Correlação entre faciologia e biodegradação dos arenitos asfálticos da Formação Piramboia, Bacia do Paraná. Dissertação de Mestrado, Universidade Estadual do Norte Fluminense.

Marynowski L., Zaton M., Simoneit B.R., Otto A., Jedrysek M.O., Grelowski C., Kurkiewicz S. 2007. Compositions, sources and depositional environments of organic matter from the Middle Jurassic clays of Poland. Applied Geochemistry, 11:2456-2485. 
Milner C., Rogers M., Evans C. 1977. Petroleum transformations in reservoirs. Journal of Geochemical Exploration, 7:101-153.

Moldowan J.M., Seifert W.K., Gallegos E.J. 1985. Relationship between petroleum composition and depositional environment of petroleum source rocks. AAPG Bulletin, 69:1255-1268.

Moldowan J.M., Lee C.Y., Sundararaman P. 1992. Biological markers in sediments and petroleum. Prentice-Hall, Englewood Cliffs, New Jersey.

Palmer S. 1983. Porphyrin distributions in degraded and nondegraded oils from Columbia. In: Abstracts 186th American Chemical Society National Convention, Geochemistry Division, August 1993, Washington, DC.

Peng P.A., Morales-Izquierdo A., Hogg A., Strausz O.P. 1997. Molecular structure of Athabasca asphaltene: sulfide, ether, and ester linkages. Energy or Fuels, 11:1171-1187.

Peng P., Morales-Izquierdo A., Lown E.M., Strausz O.P. 1999. Chemical structure and biomarker content of Jinghan asphaltenes and kerogens. Energy \& fuels, 2:248-265.

Peters K.E., Moldowan J.M. 1993. The Biomarker Guide: Interpreting molecular fossil in petroleum and ancient sediments. Prentice Hall, Englewood Clifts, New Jersey.

Peters K.E., Walters C.C.C., Moldowan J.M. 2005. The biomarker guide: Biomarkers and isotopes in the environment and human history. Vol. 1, $2^{\mathrm{a}}$ ed. Cambridge University Press, Cambridge.

Seidl P.R., Chrisman E.C.A.N., Carvalho C.C.V., Leal K.Z., de Menezes S.M.C. 2004. NMR analysis of asphaltenes separated from vacuum residues by selected solvents. Journal of dispersion science and technology, 25:349-353.

Seifert W.K., Moldowan J.M. 1979. The effect of biodegradation on steranes and terpanes in crude oils. Geochimica et Cosmochimica Acta, 1:111-126.

Speight J.G. 1994. Chemical and physical studies of petroleum asphaltenes. Developments in petroleum science, 40:7-65.

Sun Y., Chen Z., Xu S., Cai P. 2005. Stable carbon and hydrogen isotopic fractionation of individual $\mathrm{n}$-alkanes accompanying biodegradation: evidence from a group of progressively biodegraded oils. Organic Geochemistry, 36:225-238.

Thomaz Filho A., Mizusaki A.M.P., Antonioli L. 2008. Magmatismo nas bacias sedimentares brasileiras e sua influência na geologia do petróleo. Revista Brasileira de Geociências, 38:128-137.

Tissot B.P. \& Welte D.H. 1984. Petroleum formation and occurrence. SpringerVerlag, $699 \mathrm{p}$.

Trigüis J.A. 1986. An Organic Geochemistry Investigation of Heat - Effected Sediments in the Paraná Basin (Brazil). Ph.D Thesis, Department of Geology, University of New Castle, 203 p. 